\title{
Development of a Hybrid Dextrin Hydrogel Encapsulating Dextrin Nanogel As Protein Delivery System
}

\author{
Maria Molinos, ${ }^{\dagger}$ Vera Carvalho, ${ }^{\dagger}$ Dina M. Silva, and Francisco M. Gama* \\ IBB, Institute for Biotechnology and Bioengineering, Centre of Biological Engineering, Universidade do Minho, Campus de Gualtar, \\ 4710-057, Braga, Portugal
}

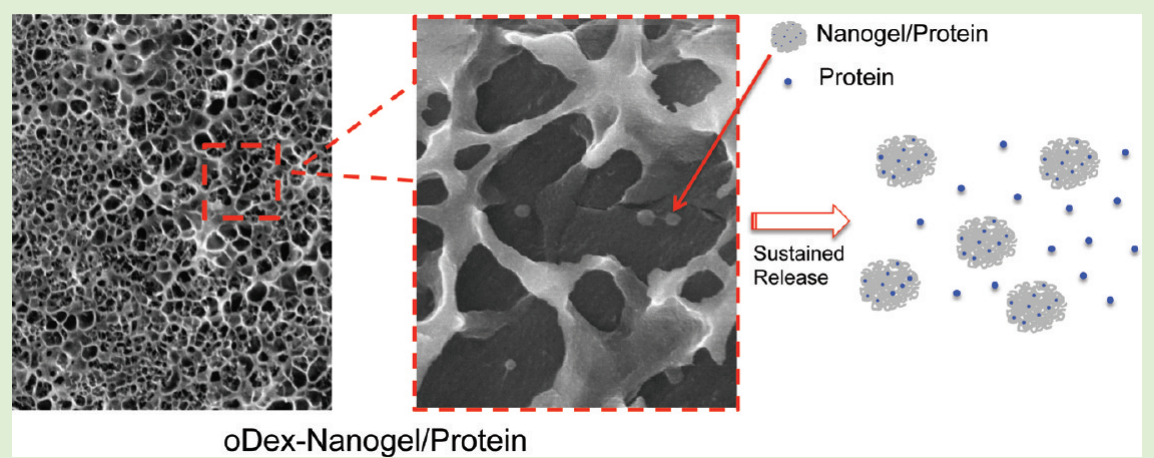

ABSTRACT: Dextrin, a glucose polymer with low molecular weight, was used to develop a fully resorbable hydrogel, without using chemical initiators. Dextrin was first oxidized (oDex) with sodium periodate and then cross-linked with adipic acid dihidrazide, a nontoxic cross-linking molecule. Furthermore, a new bidimensional composite hydrogel, made of oxidized dextrin incorporating dextrin nanogels (oDex-nanogel), was also developed. The oDex hydrogels showed good mechanical properties and biocompatibility, allowing the proliferation of mouse embryo fibroblasts 3T3 cultured on top of the gel. The gelation time may be controlled selecting the concentrations of the polymer and reticulating agent. Both the oDex and oDex-nanogel hydrogels are biodegradable and present a 3-D network with a continuous porous structure. The obtained hybrid hydrogel enables the release of the dextrin nanogel over an extended period of time, paralleling the mass loss curve due to the degradation of the material. The dextrin nanogel allowed the efficient incorporation of interleukin-10 and insulin in the oDex hydrogel, providing a sophisticated system of controlled release. The new hydrogels present promising properties as an injectable carrier of bioactive molecules. Both proteins and poorly water-soluble low-molecular-weight drugs are efficiently encapsulated in the nanogel, which performs as a controlled release system entrapped in the hydrogel matrix.

\section{INTRODUCTION}

Therapeutic proteins are becoming available for the treatment of a wide range of diseases, among others cancer, autoimmune diseases, and metabolic disorders. A main problem limiting the efficiency of protein therapeutics is the reduced stability and short circulation half-lives after parenteral administration (i.e., intravenous, intramuscular, or subcutaneous). ${ }^{1}$ As a result of the invasive nature, injectable formulations are frequently faced with patient discomfort and noncompliance. In the case of proteins, susceptibility to proteolysis and colloidal instability are additional difficulties. Consequently, a high drug concentration or a high dosing frequency becomes necessary, which may lead to adverse side effects. ${ }^{1}$ Therefore, protein delivery systems are urgently needed for the enhancement of the biopharmaceuticals bioavailability and selectivity, enabling a targeted controlled release profile. Aiming at achieving an effective protein delivery, carriers such as liposomes, polymer micelles, and hydrogels (micro- and nano-hydrogels) have been developed. ${ }^{1-4}$

Hydrogels are used in clinical practice and experimental medicine in diverse applications, including tissue engineering and as drug delivery systems. ${ }^{5,6}$ They can be achieved through chemical or physical cross-linking of polymers (natural or synthetic), their properties depending on the chemical composition, crosslinking density, and hydrophobicity. Hydrogels are commonly considered as highly biocompatible, owing to the high water content and also to the physiochemical similarity with the native extracellular matrix. Starch-based polymers, as dextrin, are examples of natural polymers with great potential for the development of hydrogels owing to its proven clinical tolerability and efficient absorption due to degradation by amylases. $^{7,8}$ Recently, Carvalho et al. ${ }^{9,10}$ produced dextrin hydrogels, namely, dextrin-vinyl acrylate (Dex-VA) and dextrinhydroxyethylmethacrylate (Dex-HEMA), and verified their noncytotoxicity as well as their appealing diffusivity and degradability profiles for targeted delivery therapeutics. Moreover, Gonçalves et al. developed and characterized a nanogel obtained by self-assembling of hydrophobized dextrin. ${ }^{11,12}$ The nanogel obtained has high colloidal stability and spherical

Received: November 10, 2011

Revised: January 11, 2012

Published: January 30, 2012 
shape. In previous work, ${ }^{13}$ it was shown that dextrin nanogels effectively incorporated and stabilized a recombinant mutated interleukin-10 (rIL-10), allowing for the release of biologically active rIL-10 over time.

However, hydrogels present some limitations regarding drug delivery: the high water content and large pore sizes frequently result in relatively rapid release. In the past decade, composite systems where micro- or nano-hydrogels are incorporated in a bulk hydrogel matrix appeared as a platform for drug delivery. ${ }^{14-17}$ The micro- or nano-hydrogel particles can act as a drug reservoir from which release can be triggered by a suitable stimulus or simply released in a diffusion-controlled manner. Simultaneous diffusion of different molecules at different rates can be obtained from the same platform by adding two (or more) populations of micro or nanogels, each loaded with one kind of drug, in the same hydrogel matrix. ${ }^{15,17}$ The major advantage relies on the improvement of the kinetic release profile of the drug, as the nanogel phase provides an additional diffusion barrier moderating or eliminating the initial burst release typically observed in hydrogel or nanogel drug delivery systems.

In this work, an expeditious methodology initially developed by Bouhadir and colleagues ${ }^{18}$ was used to prepare degradable injectable hydrogels from oxidized dextrin (oDex) without the use of any chemical initiator. The networks were evaluated regarding their rheological properties as well their degradation at physiologic $\mathrm{pH}$. A composite system made from dextrin nanogel and oDex hydrogel is also described. Their degradation and nanogel release profile were also evaluated. Simultaneously, a recombinant interleukin-10 (rIL-10), an immunoregulatory cytokine with strong anti-inflammatory activity, ${ }^{19,20}$ and insulin (Ins), an hormone responsible for controlling the cellular uptake, utilization, and storage of glucose, ${ }^{21,22}$ were used to evaluate the potential of these hydrogels as controlled protein release systems.

\section{EXPERIMENTAL SECTION}

Materials. All reagents used were of laboratory grade and purchased from Sigma-Aldrich, unless stated otherwise. Dextrin Koldex 60 starch was a generous gift from Tate \& Lyle. All other chemicals and solvents used in this work were of the highest purity commercially available.

Preparation of oDex Hydrogels. Dextrin Oxidation. In brief, aqueous solutions of dextrin $(2 \% \mathrm{w} / \mathrm{v})$ were oxidized with a $2 \mathrm{~mL}$ sodium $m$-periodate solution, with different concentrations to yield the desired theoretical degree of oxidation $\left(\mathrm{DO}_{\mathrm{t}}\right)$, at room temperature, with stirring, and in the dark. After $20 \mathrm{~h}$, the oxidation reaction was stopped by adding dropwise an equimolar amount of diethyleneglycol to reduce any unreacted periodate. The resulting solution was dialyzed for 3 days against water using a dialysis membrane with a molecular weight cutoff of $1000 \mathrm{Da}$ and then lyophilized for 10 days.

Determination of Aldehyde Groups by ${ }^{1} H$ NMR Analysis. The degree of oxidation (DO) of oxidized dextrin (oDex) is defined as the number of oxidized residues per 100 glucose residues and was quantified using the tert-butylcarbazate $(\mathrm{tBC})$ method as described elsewhere. ${ }^{18,23-26}$ The ${ }^{1} \mathrm{H}$ NMR spectra were used to determine DO, calculated as a peak area ratio in the NMR spectra according to the equation: DO $(\%)=(X / Y) \times 100$ where, $X$ is the average integral corresponding to the peak at $\delta 7.3$ and $Y$ is the average integral of the anomeric protons at $\delta 4.8$ and $\delta 5.4$.

Preparation of oDex-ADH Hydrogels. oDex was dissolved in PBS buffer (phosphate-buffered saline) $(30 \% \mathrm{w} / \mathrm{v})$ at room temperature, and an adipic acid dihydrazide $(\mathrm{ADH})$ solution (prepared separately) was added at different concentrations $(5,15$, and $30 \%$ in molar base, taking into account the number of glucose residues in the original dextrin). The cross-linking reaction was allowed to proceed during $2 \mathrm{~h}$.
The material was considered as gelified when it stopped slipping along a $90^{\circ}$ inclined surface.

Preparation of oDex-Nanogel Hydrogels. Dextrin nanogel was prepared as described in Carvalho et al. ${ }^{13}$ The nanogel formation was confirmed by dynamic light scattering, as previously described. ${ }^{11-13}$ oDex, DO $40 \%,(30 \% \mathrm{w} / \mathrm{v})$ was dissolved in PBS or in a suspension of nanogel for $\sim 16 \mathrm{~h}$ at room temperature. Then, the oDex suspensions were mixed with $5 \%$ (in molar base taking into account the number of glucose residues in the original dextrin) $\mathrm{ADH}$. The cross-linking was allowed to proceed at room temperature for $\sim 2 \mathrm{~h}$. Ahead, the oxidized dextrin hydrogels are termed as oDex hydrogels, and the oxidized dextrin hydrogels with incorporated dextrin nanogels are called oDexnanogel hydrogels.

Mechanical Analysis. The mechanical properties of cross-linked oDex hydrogels were assessed using a Mechanical Tester - ShimadzuAG-IS $1 \mathrm{kN}$ testing instrument. Each hydrogel disk (superficial area $=$ $133 \mathrm{~mm}^{2}$ ) was placed between two parallel metallic circumferential plates so that the compressive force would be uniform along the sample and compressed at room temperature with a constant deformation rate of $0.5 \mathrm{~mm} \mathrm{~min}^{-1}$. For each condition, samples in triplicate were analyzed; the values given in the results section represent the mean and the standard deviation.

Biocompatibility Assays. Mouse Embryo Fibroblasts 3T3 Culture. Mouse embryo fibroblasts 3T3 (ATCC CCL-164) were grown in Dulbecco's modified Eagle's media supplemented with $10 \%$ newborn calf serum (Invitrogen, U.K.) and $1 \mu \mathrm{g} / \mathrm{mL}$ penicillin/ streptavidin (cDMEM) at $37{ }^{\circ} \mathrm{C}$ in a $95 \%$ humidified air containing $5 \% \mathrm{CO}_{2}$. At $80 \%$ confluency, 3T3 fibroblasts were harvested with $0.05 \%$ $(\mathrm{w} / \mathrm{v})$ trypsin-EDTA and subcultivated in the same medium.

In Vitro Cytotoxicity Studies. The cytotoxicity of the un-crosslinked macromonomer solutions (oDex and $\mathrm{ADH}$ ) and hydrogel degradation extracts was assessed in a 3T3 fibroblasts culture previously incubated for $24 \mathrm{~h}\left(2.5 \times 10^{3}\right.$ cells/well, in a 96-well polystyrene plate) using 3-[4,5-dimethylthiazol-2-yl]-2,5-diphenyl tetrazolium bromide (MTT) assay, as briefly described below.

The oDex and $\mathrm{ADH}$ were sterilized by ethylene oxide (ETO) and filtration, respectively, and dissolved in cDMEM. Increasing concentrations of oDex and $\mathrm{ADH}$ were then added to the fibroblast culture. The degradation extracts were obtained immersing oDex hydrogels in cDMEM and incubating for $48 \mathrm{~h}$ at room temperature. Subsequently, the degradation extracts were added to the fibroblast culture. After $48 \mathrm{~h}$ of incubation, cell viability was accessed by MTT assay.

To evaluate the direct cytotoxicity of the oDex hydrogels toward fibroblasts, we used the live and dead assay (Invitrogen, U.K.). Mousse embryo fibroblasts $3 \mathrm{~T} 3$ were seeded $\left(5 \times 10^{4}\right.$ cells/well $)$ in a six-well polystyrene plate and incubated for $24 \mathrm{~h}$. Then, the culture medium was removed, and hydrogel discs $(\varnothing 4 \mathrm{~mm}, 2 \mathrm{~mm}$ thickness) were placed on the wells, in direct contact with cells. At regular time intervals, $200 \mu \mathrm{L}$ of a solution of $2 \mu \mathrm{M}$ calcein $\mathrm{AM}$ and $4 \mu \mathrm{M}$ ethidium homodimer-1, in sterile PBS, were added to the wells, incubated for 30 to $45 \mathrm{~min}$ at $37{ }^{\circ} \mathrm{C}, 5 \% \mathrm{CO}_{2}$ (as indicated by the manufacturer) and visualized in a fluorescence microscope. Latex discs and agar gel (U.S. Pharmacopeia) were used as controls. Morphological evaluation of 3T3 cells was also made by regular light microscope observations.

Cryo-Scanning Electron Microscopy (Cryo-SEM) Analysis. The topography and porosity of the hydrogels were studied by CryoSEM. The oDex and oDex-nanogel hydrogels (30\% w/v oDex, $10 \mathrm{mg} / \mathrm{mL}$ nanogel) were rapidly immersed in liquid nitrogen slush at $-210{ }^{\circ} \mathrm{C}$ for $2 \mathrm{~min}$ and vacuum transferred to an Alto 2500 (Gatan, Pleasanton, CA) cryo-preparation chamber attached to a JEOL JSM 6301F scanning electron microscope. Frozen samples were fractured at $-185^{\circ} \mathrm{C}$, etched during $90 \mathrm{~s}$ (to sublimate water partially from the fractured hydrogel surface), and finally gold-coated for $2 \mathrm{~min}$. Samples were viewed at $-130{ }^{\circ} \mathrm{C}$, and the resulting SEM images were analyzed using Image software (National Institutes of Health, U.S.)

Degradation of oDex Hydrogels. After being prepared and weighted $\left(W_{\mathrm{i}}\right)$, the hydrogels were immersed in PBS (diffusion medium) and incubated at $37{ }^{\circ} \mathrm{C}$. At regular intervals, they were removed from the solutions, blotted with filter paper, weighed $\left(W_{\mathrm{t}}\right)$, 
and returned to the same container. The buffer solution was replaced at each measurement, and the old one was stored for further analysis. The percentage of mass loss was determined using the equation: mass loss $(\%)=100-\left[\left(W_{\mathrm{t}} / W_{\mathrm{i}}\right) \times 100\right]$

Release Assays. Nanogel/FITC. To obtain dextrin nanogels labeled with FITC (nanogel/FITC), a nanogel solution was formed by dissolving $10 \mathrm{mg}$ of dextrin-VMA-SC ${ }_{16}$ (VMA: vinyl methacrylate; $\mathrm{SC}_{16}$ : alkyl chain) in $1.3 \mathrm{~mL}$ of $0.1 \mathrm{M}$ sodium phosphate buffer $\mathrm{pH} 7$ and stirred for $30 \mathrm{~min}$. Simultaneously, a fluorescein solution was prepared by dissolving $5 \mathrm{mg}$ of SAMSA (5-(2-(and-3)-S(acetylmercapto)succinoyl) amino) fluorescein (Invitrogen, U.K.) in $0.5 \mathrm{~mL}$ of $0.1 \mathrm{M} \mathrm{NaOH}$ and stirred for $15 \mathrm{~min}$. Then, $7 \mu \mathrm{L}$ of $6 \mathrm{M}$ $\mathrm{HCl}$ and $0.1 \mathrm{~mL}$ of $0.5 \mathrm{M} \mathrm{NaPO}_{4}$ buffer $\mathrm{pH} 7$ were added and stirred for $10 \mathrm{~min}$. Finally, the nanogel solution and the fluorescein solution were mixed and stirred for $30 \mathrm{~min}$. Unbound FITC was separated using a Sephadex G25 PD10 column (Amersham Biosciences) equilibrated with PBS, and the labeled nanogel (nanogel/FITC) was eluted with PBS.

The oDex-nanogel/FITC hydrogel was obtained as previously described (preparation of oDex-nanogel hydrogel). Degradation assays were also performed, as described above. The nanogel/FITC release from the oDex-nanogel hydrogels was evaluated by fluorimetry. The fluorescence intensity of the supernatant collected from the degrading hydrogels, at regular intervals, was measured using a spectrofluorimeter (Fluorolog Horiba Jobin Yvon). Fluorescence spectra were collected using an excitation wavelength of $460 \mathrm{~nm}$ and recording emission between 470 and 600 at $1 \mathrm{~nm}$ intervals. The slit width was set at $6.0 \mathrm{~nm}$ for the excitation and emission. The fluorescence intensity was measured at the maximum of the peak obtained $(520 \mathrm{~nm})$.

The percentage of nanogel/FITC released from the oDex-nanogel hydrogel was obtained according to the equation: Nanogel/FITC release $(\%)=[\text { nanogel } / \text { FITC }]_{\text {det }} /[\text { nanogel } / \text { FITC }]_{\text {tot }} \times 100$, where $[\text { nanogel/FITC }]_{\text {det }}$ is the fluorescence intensity detected in the PBS supernatant collected during degradation and $[\text { nanogel/FITC }]_{\text {tot }}$ is the fluorescence intensity obtained dispersing the nanogel/FITC in the same volume as that used in the degradation assays.

Nanogel/rlL-10. The nanogel/rIL-10 complex was obtained by dissolving the lyophilized dextrin-VMA-SC ${ }_{16}$ in a rIL-10 solution ( $5 \mathrm{mg} / \mathrm{mL}$ nanogel, $25 \mu \mathrm{g} / \mathrm{mL}$ rIL-10), as previously described. ${ }^{13}$ The oDex-nanogel/rIL-10 hydrogel was then prepared as already explained. The oDex hydrogel with incorporated free rIL-10 was obtained by dissolving oDex in a rIL-10 solution $(25 \mu \mathrm{g} / \mathrm{mL})$.

The rIL-10 release from the oDex hydrogels, after being immersed in PBS with $10 \%$ FBS and incubated at $37{ }^{\circ} \mathrm{C}$, was evaluated by quantifying by enzyme-linked immunosorbent assay (ELISA), the free rIL-10 in the diffusion medium. The percentage of rIL-10 released was obtained by the following equation: rIL-10 release $(\%)=[\mathrm{rIL}-10]_{\mathrm{det}} /$ $[\mathrm{rIL}-10]_{\text {tot }} \times 100$, where $[\mathrm{rIL}-10]_{\operatorname{det}}$ is the amount of rIL-10 detected in the diffusion medium at determined time and [rIL-10] $]_{\text {tot }}$ is the total amount of rIL-10 entrapped in the dextrin nanogel or in the hydrogel.

Circular Dichroism (CD) Measurements of rlL-10. The secondary structure of rIL-10 was investigated using CD. rIL-10 stability $(0.25 \mathrm{mg} / \mathrm{mL}$ in PBS $)$ at $37^{\circ} \mathrm{C}$ was accessed by obtaining CD spectra of the sample at determined intervals of time. Spectra were obtained as described in Carvalho et al. ${ }^{13}$

Nanogel/Ins-FITC. To obtain Ins labeled with FITC (Ins-FITC), we prepared a $5 \mathrm{mg} / \mathrm{mL}$ Ins solution in $0.1 \mathrm{M}$ sodium carbonate buffer $\mathrm{pH}$ 9.3. Simultaneously, a $0.04 \mathrm{mg} / \mathrm{mL}$ fluorescein isothiocyanate isomer I (where referred as FITC) solution was prepared in $0.1 \mathrm{M}$ sodium carbonate buffer $\mathrm{pH}$ 9.3. Then, for each milligram of Ins, $20 \mu \mathrm{g}$ of FITC was added, mixed, and incubated overnight at $4{ }^{\circ} \mathrm{C}$. Unbound FITC was separated using a Sephadex G25 PD10 column (Amersham Biosciences) equilibrated with PBS, and the labeled Ins (Ins-FITC) was eluted with PBS.

The nanogel/Ins-FITC complex was obtained by dissolving the lyophilized dextrin-VMA-SC ${ }_{16}$ in an Ins-FITC solution $(1 \mathrm{mg} / \mathrm{mL}$ nanogel, $0.1 \mathrm{mg} / \mathrm{mL}$ Ins-FITC). The oDex-nanogel/Ins-FITC hydrogel was then prepared as described. The oDex hydrogel with incorporated free Ins-FITC was obtained by dissolving oDex in an Ins-FITC solution $(0.1 \mathrm{mg} / \mathrm{mL})$.
The Ins-FITC release from the oDex hydrogels, after being immersed in PBS and incubated at $37{ }^{\circ} \mathrm{C}$, was evaluated by fluorimetry. The fluorescence intensity of the supernatant collected from the degrading hydrogels, at regular intervals, was measured using a Synergy HT MultiMode Microplate Reader (BioTek Instruments, Winooski, VT). Fluorescence intensities were obtained using an excitation wavelength of 485/20 nm and recording emission at 528/20 nm. All data were collected with KC4 software (BioTek Instruments, Winooski, VT).

The percentage of Ins-FITC released from the oDex-nanogel hydrogel was obtained according to equation: Ins-FITC release (\%) = $[\text { Ins-FITC }]_{\operatorname{det}} /[\text { Ins-FITC }]_{\text {tot }} \times 100$, where $[\text { Ins-FITC }]_{\operatorname{det}}$ is the amount of Ins-FITC detected in the diffusion medium at determined time and [Ins-FITC $]_{\text {tot }}$ is the total amount of Ins-FITC entrapped in the dextrin nanogel or in the hydrogel.

Data Analysis. Data are presented as means \pm SD of the indicated number $(n)$ of assays. Statistical analysis was performed using the variance analysis method (one-way ANOVA). Significant differences between samples were determined through the Dunnet test.

\section{RESULTS AND DISCUSSION}

Characterization of oDex. The periodate oxidation of carbohydrates is widely known and routinely used as a tool for elucidating the structural features of polysaccharides. ${ }^{27,28}$ In homopolysaccharides, such as dextrin, this oxidation reaction is characterized by the specific cleavage of the $\mathrm{C} 2-\mathrm{C} 3$ linkage of glucopyranoside rings, yielding two aldehyde groups per glucose unit $^{26,29}$ (Scheme 1).

The quantification of aldehyde groups, that is, oxidation degree (DO), was performed using $\mathrm{tBC}$, as previously described by Jia et al., Bouhadir et al., and Maia et al. ${ }^{18,23-26}$ Carbazates are well-known to react with aldehydes to form stable carbazones in a similar manner to hydrazone formation, making it possible to determine the aldehyde content of dextrin by ${ }^{1} \mathrm{H}$ NMR spectroscopy analysis.

Figure 1 depicts a typical ${ }^{1} \mathrm{H}$ NMR spectrum obtained for $25 \%$ oxidized dextrin (oDex 25\%). The peaks between $\delta 4.0$ and $\delta$ $3.4 \mathrm{ppm}$ are assigned to protons at positions $2,3,4,5$ and 6 , whereas the peak at $\delta 5.4$ is attributed to the glucose anomeric proton. The spectrum also shows a small peak at $\delta 5.3$ corresponding to the anomeric proton corresponding to $\alpha-1,6$ linkages $\left(<5 \%\right.$ of the total dextrin $\left.{ }^{8}\right)$. The three peaks between $\delta 7.4$ and $\delta 7.2$ are assigned to the proton attached to the carbon with linked $\mathrm{tBC}$, and the singlet at $\delta 1.5$ is assigned to $\mathrm{tBC}$.

The degree of oxidation of oDex can be easily controlled by the relative quantity of sodium periodate used, yielding free aldehyde reactive groups to create covalent linkages with reticulating molecules (e.g., $\mathrm{ADH}$ ) as well as with cellular adhesion binding peptides [as glycine-arginine-glycine-aspartic acid-tyrosine (GRGDY) peptide ${ }^{18}$ ] or even with specific drugs for targeted controlled delivery systems.

Preparation and Characterization of oDex Hydrogels. The $\mathrm{ADH}$ hydrazide groups react with the oxidized dextrin forming a network of hydrolyzable hydrazone bonds (Scheme 1). Once these covalent bonds are cleaved, hydrazides and dextrin can easily diffuse across human tissues and be eliminated via renal clearance, owing to their low molecular weight (degree of polymerization of $\sim 13^{30}$ ) fairly below the renal threshold $(\sim 40000$ Da). Also, starch polymers, as dextrin, can be hydrolyzed enzymatically by $\alpha$-amylase, which is one of the enzymes responsible for in vivo degradation of dextrin. ${ }^{8,31}$ The solubility pattern of oDex was accessed. It was noticed that above $30 \%(\mathrm{w} / \mathrm{v})$ solutions were extremely viscous and practically impossible to homogenize. So, this concentration was considered to be the threshold of oDex solubility in phosphate buffer $\mathrm{pH} 6.0$ and 
Scheme 1. (A) Periodate Oxidation of Dextrin, Yielding Two Aldehyde Groups at Positions C2 and C3 of a D-Glucose Unit and (B) Polymerization Reaction of Oxidized Dextrin with ADH
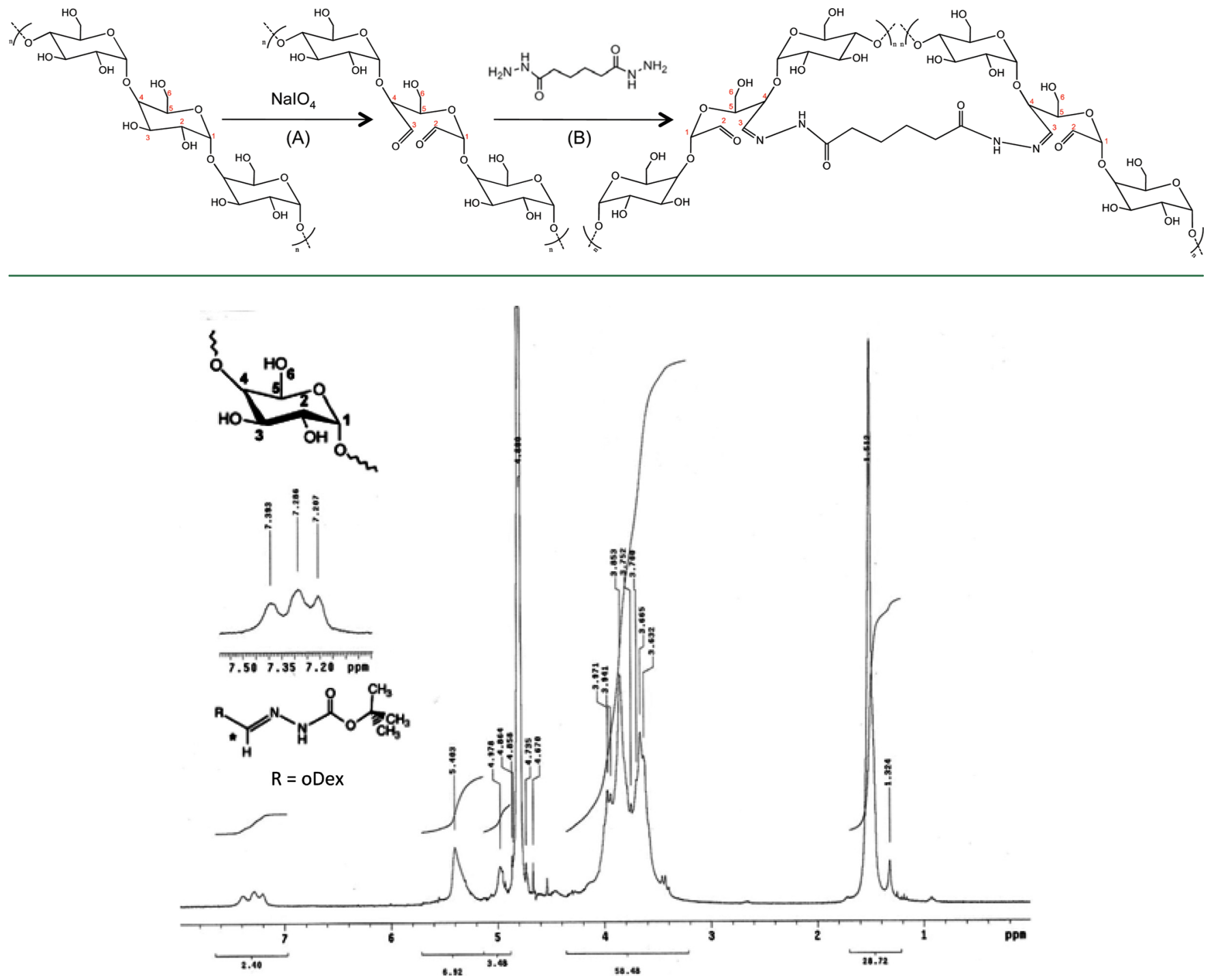

Figure 1. Oxidized dextrin (DO 25\%) ${ }^{1} \mathrm{H}$ NMR spectrum.

was further on applied in the synthesis of all oDex hydrogels. Next, oDex was cross-linked with various concentrations of $\mathrm{ADH}$. Hydrogel samples were initially produced with DOs varying from 25 to $50 \%$ and $\mathrm{ADH}$ concentrations between 5 and $30 \%$ (in molar ratio, taking into account the number of glucose residues in the original dextrin).

Table 1 shows the approximate gelation periods obtained. As expected, the cross-linking times decrease with increasing DOs as well as with increasing amounts of reticulating agent. It was found that DOs above $40 \%$ yield very viscous solutions that react promptly with $\mathrm{ADH}$, impairing good homogenization and resulting in mat and brittle hydrogels. Through control of DO and $\mathrm{ADH}$ concentrations, a good control over the gelation time is thus possible, making this hydrogel suitable as an injectable system.

According to Anseth et al., ${ }^{32}$ the compressive modulus of hydrogels is directly proportional to the intermolecular crosslink density. Hence, the influence of the $\mathrm{ADH}$ concentration, $\mathrm{DO}$, and also the type of solvent used on the extent of intermolecular cross-linking was evaluated by quantifying the
Table 1. Gelation Periods Estimated for Each oDex with Different ADH Concentrations

\begin{tabular}{|c|c|c|c|}
\hline \multirow[t]{2}{*}{$\mathrm{DO}_{\mathrm{T}}(\%)^{a}$} & \multicolumn{3}{|c|}{$\operatorname{ADH}(\%)^{b}$} \\
\hline & 5 & 15 & 30 \\
\hline 25 & $+^{c}$ & + & + \\
\hline 32.5 & $++(\sim 30 \mathrm{~min})$ & $++(\sim 10 \mathrm{~min})$ & $++(\sim 5 \mathrm{~min})$ \\
\hline 40 & $++(\sim 2 \mathrm{~min})$ & +++ & +++ \\
\hline 50 & +++ & +++ & +++ \\
\hline
\end{tabular}

${ }^{a}$ Calculated as the molar ratio of sodium periodate per initial glucose unit in dextrin. ${ }^{b}$ Calculated in molar base, taking into account the number of glucose residues in the original dextrin. ${ }^{c}(+)$ Over $1 \mathrm{~h}$ of gelation, $(++)$ gelation in $<30 \mathrm{~min},(+++)$ Gelation in $<1 \mathrm{~min}$.

compressive modulus of oDex hydrogels. This approach was previously described by Bouhadir and coworkers. ${ }^{18}$ Figure 2 presents a typical compression curve obtained for oDex hydrogels, from which the compressive modulus was determined, using the equation: compressive modulus $(\mathrm{kPa})=$ (stress max/superficial area) $\times 10^{-3}$. 


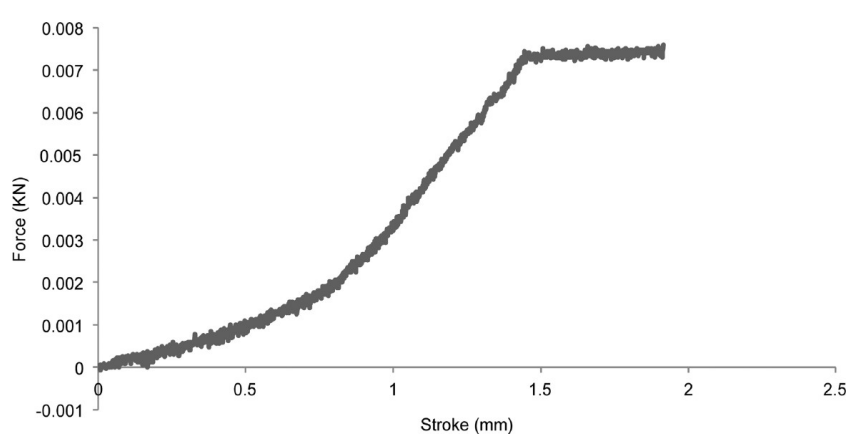

Figure 2. Compression curve showing typical behavior for an oDex DO $35 \%$ with $4 \% \mathrm{ADH}$ hydrogel.

The increasing concentration of $\mathrm{ADH}$ resulted in a proportionally higher compressive modulus of cross-linked oDex hydrogels (Figure 3A), suggesting the establishment of an increasing number of intermolecular bonds as more hydrazide groups become available to react. The same tendency was reported by Maia et al. ${ }^{25}$ with dextran hydrogels and by Bouhadir et al. ${ }^{18}$ with poly(aldehyde guluronate) hydrogels, the former revealing inferior compressive strength even with higher concentrations of reticulating agent. In fact, the maximum compressive modulus obtained with guluronate hydrogels was $560 \mathrm{kPa}$, using $150 \mathrm{mM} \mathrm{ADH}$, whereas with ca. $130 \mathrm{mM}$ (equivalent to $10 \%$ in molar ratio) a compressive modulus of $600 \mathrm{kPa}$ for oDex $35 \%$ hydrogels was registered. Thus, dextrin hydrogels appear to have better mechanical properties.

The degree of oxidation does not seem to play a major influence on the intermolecular cross-links. Interestingly, oDex DO $25 \%$ hydrogels revealed the maximum compressive force (c.a. $533 \mathrm{kPa}$ ), although there was no significant difference $(p>0.05)$ relatively to higher DO oDex hydrogels (Figure 3B). With $25 \%$ oxidation there is an average two to three oxidized glucose residues per dextrin molecule (taking into account the average polymerization degree of dextrin, which is ca. 10-13). Hence each molecule must participate in two distinct bonds with different molecules to be efficiently reticulated. The excessive modification of the original polymeric chain, making available a greater number of reactive oxidized groups, does not necessarily imply an enhancement on hydrogel's mechanical properties, maybe due to steric rearrangement impediment that avoids the formation of a larger number of intermolecular bonds.

Injectable hydrogels should be able to proceed its polymerization process in situ, meaning the interstitial fluids or blood should not interfere with it, for instance, by influence of the media $\mathrm{pH}$. Also, the intrinsic conditions necessary for the hydrogel's formation must not be harmful to the surrounding tissues. Hence, the $\mathrm{pH}$ influence on the density of intermolecular bonds was evaluated by measuring the compressive modulus of various oDex hydrogels prepared in four different solvents: double-distilled water (c.a pH 5.77), 0.1 M phosphate buffer ( $\mathrm{pH}$ 6.0), PBS ( $\mathrm{pH} 7.4$ ), and cDMEM (c.a pH 7.5). The results shown in Figure 4 are in agreement with the tendency

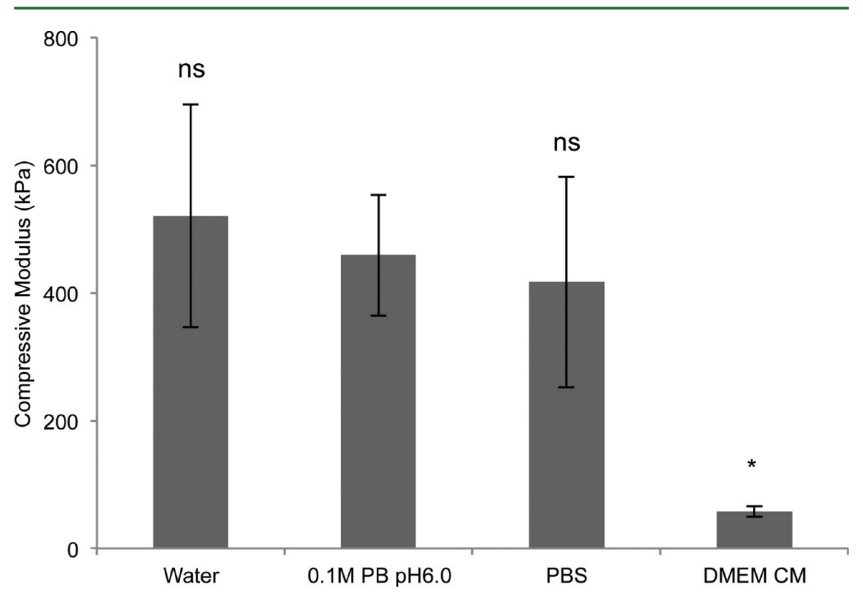

Figure 4. Compressive modulus of cross-linked oDex hydrogels as a function of the solvent in which they are prepared. All hydrogels were prepared with oDex $(30 \% \mathrm{w} / \mathrm{v})$ and $5 \% \mathrm{ADH}$. Results presented as average $\pm \mathrm{SD}(n=3)$. ns: nonsignificant, $p>0.05 ; * p<0.05$, compared with $0.1 \mathrm{M}$ phosphate buffer, $\mathrm{pH}$ 6.0.

previously verified by Bouhadir et al. ${ }^{18}$ In fact, it is well known that the reactivity of hydrazide groups with aldehydes is optimal at lower $\mathrm{pH}$ values. Under acidic conditions, the reactive aldehyde groups formed upon periodate oxidation are prone to react with the hydrazide groups, leading to hydrazone bonds. Under neutral to basic conditions, however, slower kinetics are
A

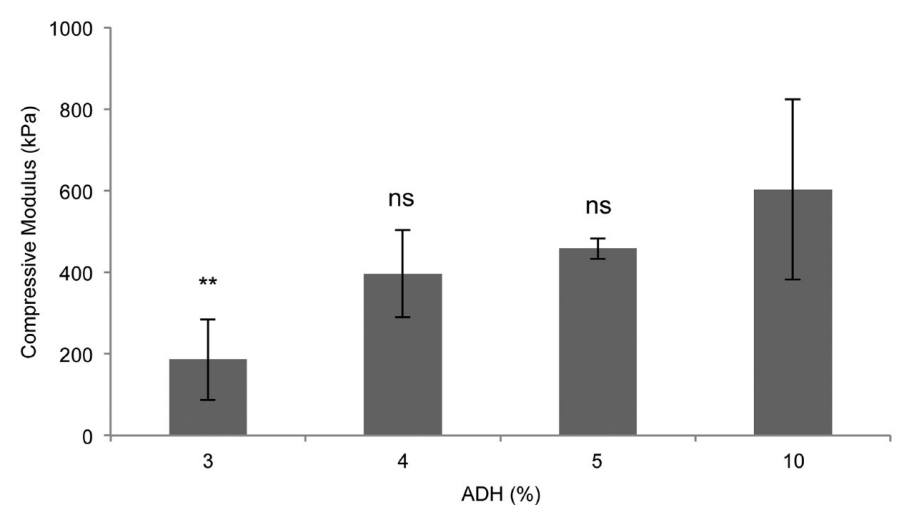

B

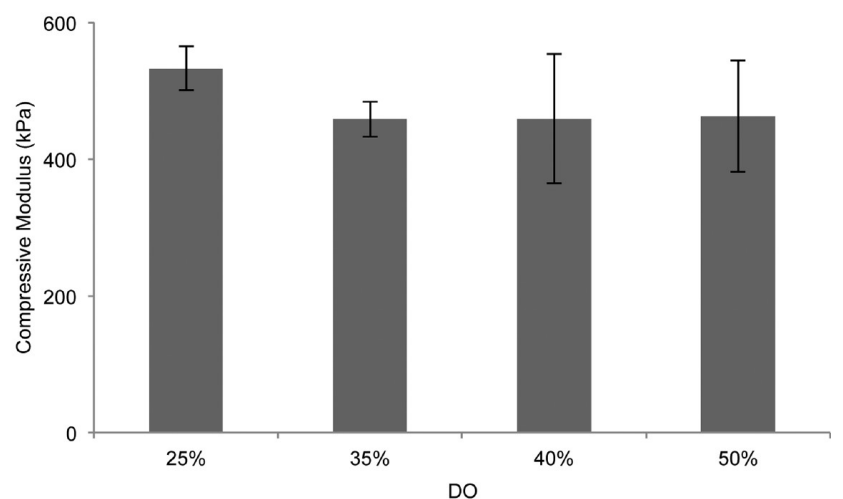

Figure 3. Compressive modulus of $(\mathrm{A})$ cross-linked oDex hydrogels as a function of the $\mathrm{ADH}$ concentration (in molar ratio, taking into account the number of glucose residues in the original dextrin, oDex DO $35 \%(30 \% \mathrm{w} / \mathrm{v})$ in $0.1 \mathrm{M}$ phosphate buffer, $\mathrm{pH} 6.0)$ and (B) cross-linked oDex hydrogels as a function of the degree of oxidation of dextrin (oDex DO 35\% and 5\% ADH in $0.1 \mathrm{M}$ phosphate buffer, $\mathrm{pH} 6.0$ ). Results presented as average $\pm \mathrm{SD}(n=3)$. ns: nonsignificant, $p>0.05$; ** $p<0.01$, compared with the highest concentration of ADH used. 
A

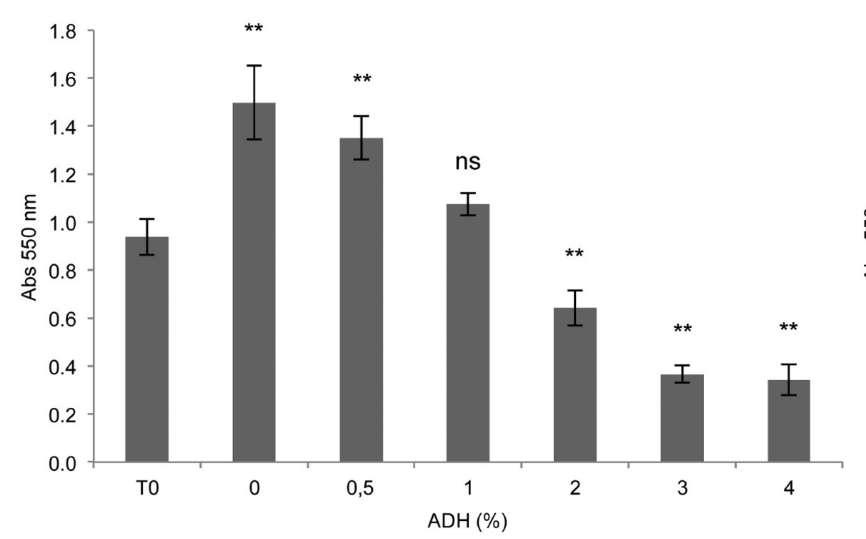

B

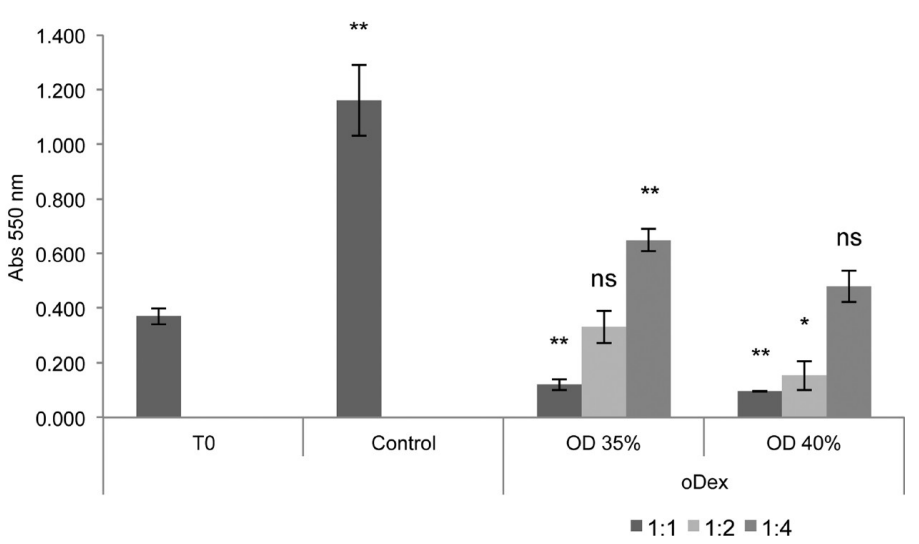

Figure 5. MTT absorbance values obtained after $48 \mathrm{~h}$ of incubation of $3 \mathrm{~T} 3$ cells (A) in direct contact with different concentrations of reticulating agent $(\mathrm{ADH})$ and $(\mathrm{B})$ with degradation products (1:1, 1:2, and 1:4 dilutions) of oDex hydrogels (T0, initial cell abs; Control, abs after $48 \mathrm{~h}$ of incubation). Results presented as average $\pm \mathrm{SD}(n=3)$. ns: nonsignificant, $p>0.05 ; *<0.05$; $* * p<0.01$, compared with the T0 control.
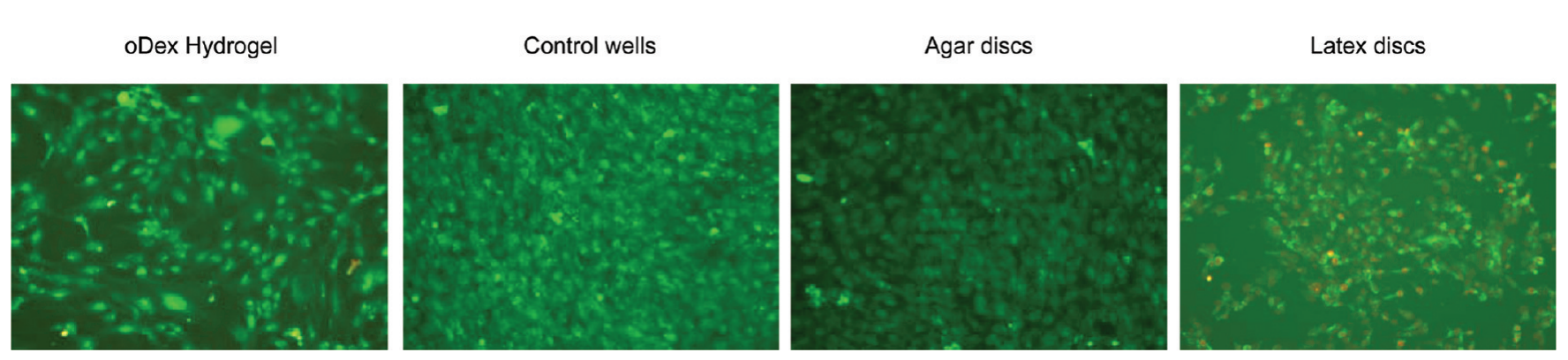

Figure 6. Fluorescence photographs of mouse embryo fibroblasts $3 \mathrm{~T} 3$ cells stained with live and dead after $48 \mathrm{~h}$ of incubation. Live cells are stained in green and dead cells are stained in red.

in effect, and a longer time is required for the completion of the reaction, yielding a lower degree of functional cross-linking. A possible explanation for the low compressive modulus values $(58 \mathrm{kPa})$ of hydrogels prepared in CDMEM, could be supported by the presence of amino acids in solution, which might be interacting with aldehyde groups in oDex, following hydrolysis of hydrazone bonds.

Biocompatibility. As to characterize the biocompatibility of the oDex hydrogels, the cytotoxicity was evaluated for un-crosslinked macromonomer solutions, cross-linked hydrogels, as well as its degradation products. Mouse embryo fibroblasts 3T3 cells were used. Cytotoxicity, defined as the "in vitro evaluation of toxicological risks using cell culture", is a way to assess the in vitro biocompatibility of materials to be used in biomedical applications. Moreover, the information gained from these types of investigations may be used in the design of further in vivo experiments.

Figure 5A depicts the MTT absorbance values obtained after $48 \mathrm{~h}$ of incubation with different concentrations of $\mathrm{ADH}$. After $48 \mathrm{~h}$ of incubation, higher concentrations of $\mathrm{ADH}(2-4 \% \mathrm{w} / \mathrm{v})$ induce cell death. However, when the amount of $\mathrm{ADH}$ used to form the oDex hydrogels (5\% molar base corresponding to $1 \% \mathrm{w} / \mathrm{v}$ ) was incubated with $3 \mathrm{~T} 3$ fibroblasts, no significant difference was noted in MTT absorbance values.

The products of the hydrogels degradation can be potentially cytotoxic; to evaluate its toxicity, we incubated the extracts obtained during degradation with mouse embryo fibroblasts 3T3 cells, and MTT assay was used to measure cellular viability. The results are shown in Figure 5B. Cellular death is observed when the oDex degradation products are in direct contact with cells, although this effect attenuates upon dilution. Cellular death could be caused by the mechanical pressure or by the diminished oxygenation and nutrient diffusion caused by the products sedimentation observed with the undiluted degradation products. A similar effect was observed for the oDex solutions tested and has also been reported by other authors (Massia and Stark ${ }^{33}$ and Ferreira et al. ${ }^{34}$ ) using different materials.

Dex-HEMA hydrogels were previously developed by Carvalho and coworkers. ${ }^{10}$ In vivo assays, performed by Moreira et al., ${ }^{30}$ showed that after implantation Dex-HEMA hydrogels were quickly and completely degraded and reabsorbed, even though in vitro studies revealed cytotoxicity associated with Dex-HEMA degradation products. Therefore, the rapid reabsorption and excretion is likely to minimize the biological impact observed in this assay.

To evaluate oDex hydrogels cytotoxicity, the live and dead assay was performed. oDex hydrogels were placed in direct contact with cells, and latex discs and agar gels were used as controls. In Figure 6, fluorescence images of mouse embryo fibroblasts 3T3 cells after $48 \mathrm{~h}$ of incubation are shown. As expected, latex discs revealed high toxicity, as the cells appear majorly red (dead cells). On the contrary, with agar discs and oDex hydrogels the majority of cells are alive (green cells). It is well-recognized that the surface chemistry of hydrogels can influence cell adhesion and proliferation. It has already been shown $^{35,36}$ that cell proliferation is sensitive to both hydrogel charge density and cross-linker concentration.

Figure 7 illustrates that the direct contact of oDex hydrogels does not inhibit cell proliferation. Although in lower number as 


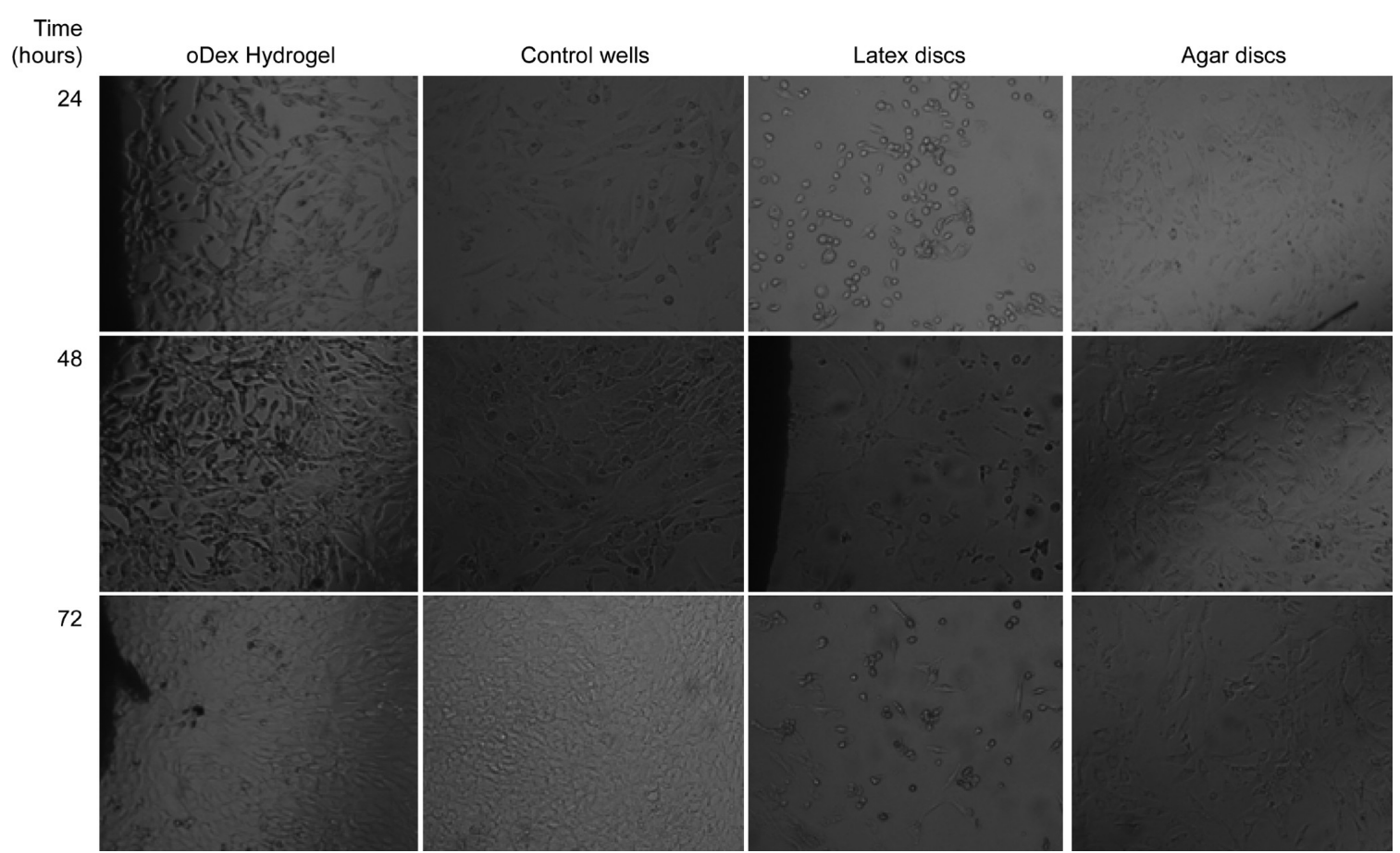

Figure 7. Morphologic evaluation of 3T3 cells in direct contact with dextrin hydrogels, cell culture plates (control wells), agarose gel, and latex rubber. (Dark shadows on the left side show part of hydrogel or latex disk.)

compared with control wells, cells are adherent and retain the typical fibroblast morphology.

The overall results point to the oDex hydrogels cytocompatibility: in direct contact with $3 \mathrm{~T} 3$ fibroblasts, the oDex hydrogels do not induce cell death nor inhibit cell proliferation under the conditions tested and for mousse embryo fibroblasts 3T3.

Cryo-SEM Analysis. The dextrin nanogel, reported in previous publications, ${ }^{12,13,37,38}$ was incorporated in the hydrogel, and the hybrid hydrogel was further characterized. The morphologies of the oDex hydrogels were examined by CryoSEM. As expected, the covalent cross-linking produced 3-D networks. As seen in Figure 8, the oDex hydrogels exhibit a continuous porous structure, with a diameter of $\sim 1 \mu \mathrm{m}$. With a larger amplification, the nanogel particles present in oDexnanogel hydrogel can be observed.

No obvious morphologic differences are noticeable comparing the oDex and oDex-nanogel hydrogel formulations. All hydrogels have random morphology and similar porous structure, and the incorporated nanogels did not have significant influence in the morphology of the oDex hydrogel network.

Degradation Kinetics of oDex Hydrogels at Physiologic $\mathrm{pH}$. Despite their many favorable properties, hydrogels also have some limitations. The low tensile strength limits their use in load-bearing applications and, as a consequence, the premature dissolution or flow away of the hydrogel from the targeted local site can occur. Concerning drug delivery, the most important drawback of hydrogels relates to the quantity and homogeneity of drug loading, which may be limited, especially in the case of hydrophobic drugs; on the other hand, the high water content and large pores frequently result in relatively rapid drug release. To surmount these limitations, a nanogel previously developed and characterized in our lab was used to produce a new bidimensional hydrogel. Because this nanogel is obtained by self-assembling of amphiphilic molecules and has been shown to incorporate and stabilize both proteins ${ }^{13}$ and small hydrophobic pharmaceuticals, ${ }^{39}$ the presence of the nanophase may be useful for the formulation of hydrogels as a controlled drug release system.

Figure 9A depicts the mass loss profile and nanogel release profile of oDex and oDex-nanogel hydrogels with different concentrations of nanogel ( 1 and $3 \mathrm{mg} / \mathrm{mL}$ ) as a function of time when incubated in PBS at $37{ }^{\circ} \mathrm{C}$.

The oDex cross-link junctions are degraded along time, leading to a correspondingly gradual swelling. When the crosslinks are hydrolyzed, the network swells, imbibing more water and leading to further hydrolysis. For both kinds of hydrogel (oDex and oDex-nanogel), there is a rapid initial mass loss, likely due to the release of material with a lower cross-linking density ( 2 to 3 days), followed by a second stage with a lower rate of solubilization. Dextrin molecules may be reticulated through 1,2 , or 3 bonds; hence, their release rate is likely to be variable.

According to mass loss studies, the degradation speed of oDex hydrogel is different from the one found in oDex-nanogel hydrogels. In approximately 25 days, the oDex hydrogel network is completely solubilized, whereas only $\sim 70 \%$ mass loss was observed in oDex-nanogel hydrogels. Only a slight difference in mass loss is observed comparing the two formulations with different amounts of nanogel.

The slower degradation rate observed in the presence of the nanogel may be assigned to a further reticulation of the hydrogel network. Zhang et al. ${ }^{40}$ described a composite system made of poly $(N$-isopropylacrylamide) (NIPAAm) hydrogel and hydroxyl-functionalized glycerol poly( $\varepsilon$-caprolactone) (PGCL)based microspheres, where the incorporation of the hydrophobic PGCL-based microspheres into the hydrogel led to greatly increased mechanical properties of the resulting networks. These improved mechanical properties were assigned to the existence of hydrophobic solid microspheres, which acted like reinforcing nodes. Nuño-Donlucas et al. ${ }^{41}$ also reported similar findings of polyacrylamide hydrogels with 

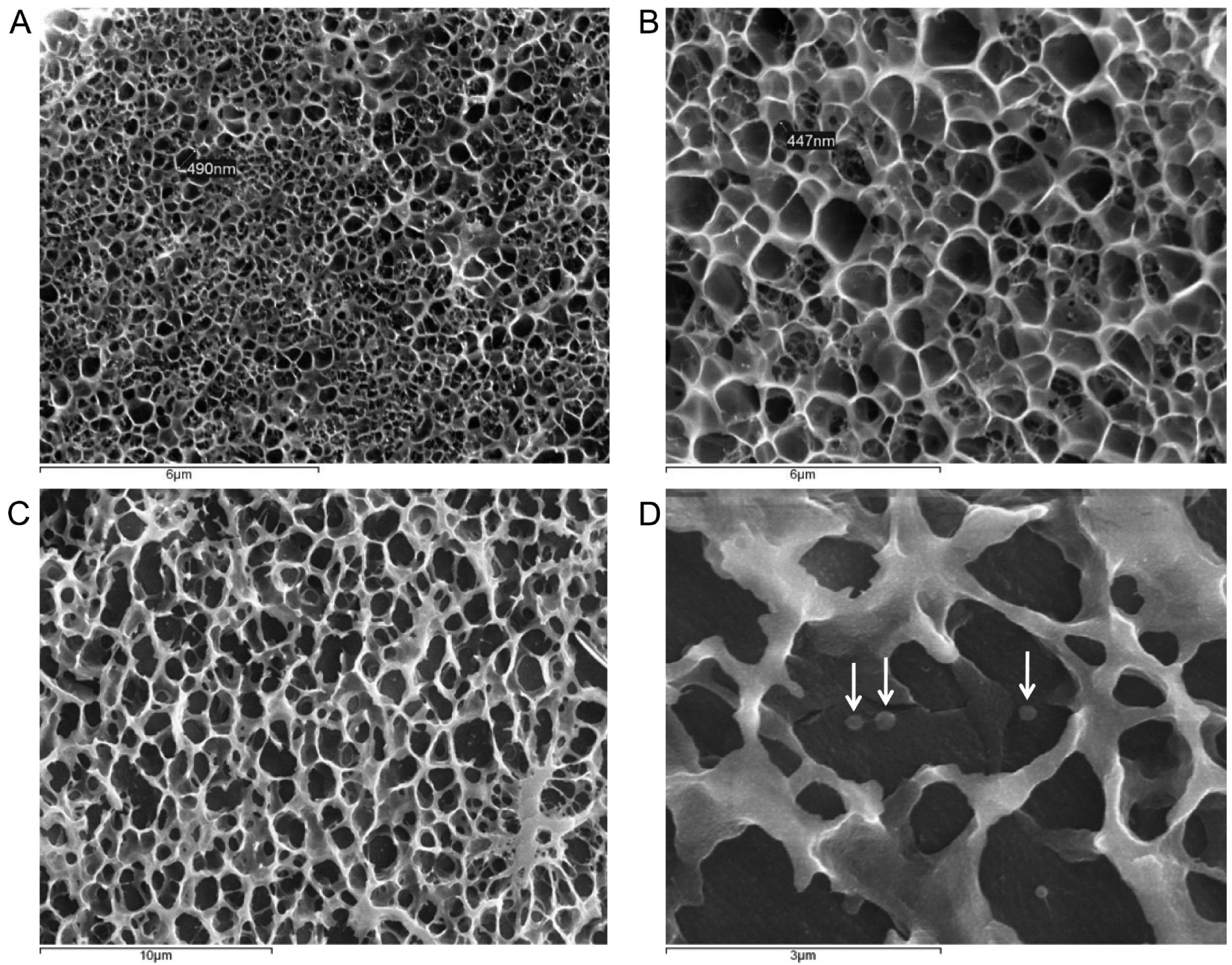

Figure 8. Cryo-SEM images from cross-section of oDex hydrogel (A) before and (B) after immersion on PBS buffer for $24 \mathrm{~h}$ and (C,D) oDexnanogel hydrogel. Arrows show the dextrin nanogels (oDex DO 35\%).

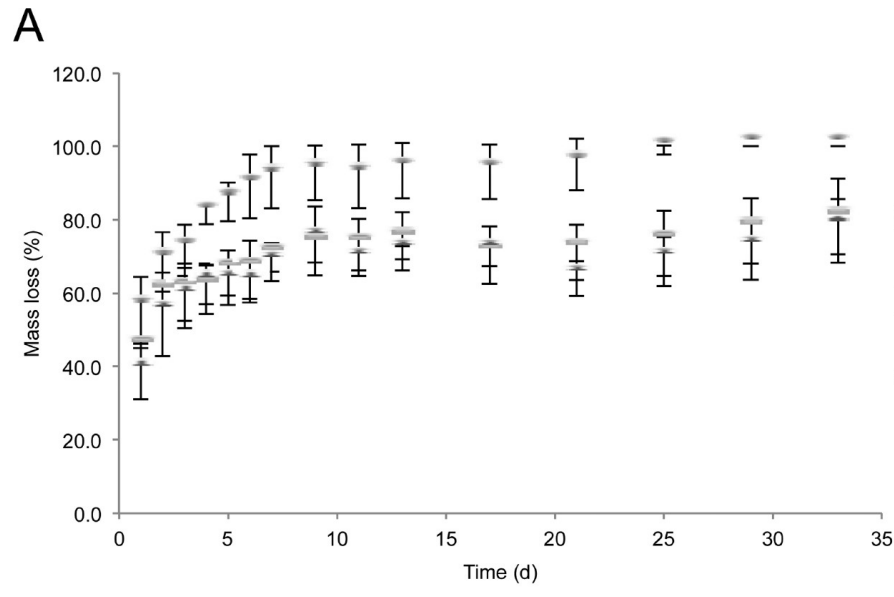

$\diamond$ oDex $\square$ oDex-Nanogel $(1 \mathrm{mg} / \mathrm{mL}) \quad \Delta$ oDex-Nanogel $(3 \mathrm{mg} / \mathrm{mL})$
B

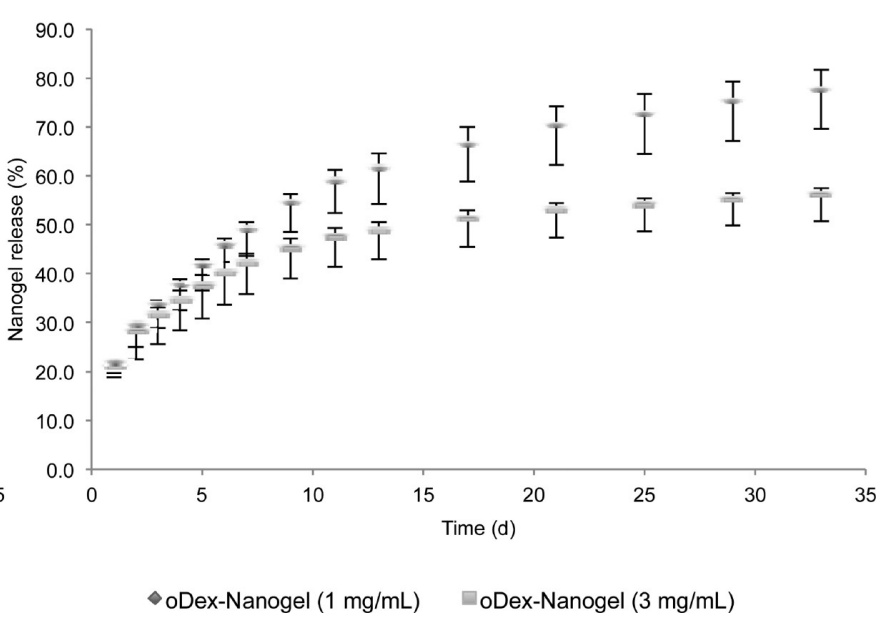

Figure 9. (A) Mass loss and (B) nanogel cumulative release profiles of oDex, oDex-nanogel $(1 \mathrm{mg} / \mathrm{mL}$ and $3 \mathrm{mg} / \mathrm{mL})$ hydrogels. Shown are mean \pm $\mathrm{SD}(n=3)$.

increased mechanical properties by incorporating poly(methyl methacrylate) nanoparticles. Additionally, Puig et al. ${ }^{42}$ presented a composite hydrogel of polyacrylamide nanoparticles in a polyacrylamide matrix with improved mechanical properties.
Depending on the chemical structure of the polymer backbone, hydrogel degradation can occur by either surface or bulk erosion. Surface erosion takes place when the rate of erosion exceeds the rate of water permeation into the bulk of the polymer. Bulk erosion occurs when water molecules permeate into the bulk of 
the matrix at a faster rate than erosion, thus exhibiting complex degradation/erosion kinetics. Most of the biodegradable polymers used in drug delivery undergo bulk erosion, similar behavior being observed in hydrogels made from biodegradable polymers. ${ }^{43}$ In oDex hydrogels, degradation mainly occurs by bulk erosion, and it is characterized by nonlinear degradation profile accompanied by an increasing pore size, as seen in Figure 8A,B. The variation in pore size during the degradation of the hydrogel network is important because it affects the swelling of the hydrogel, the diffusion of molecules, and the delivery of cells when the hydrogels are used for cell encapsulation. ${ }^{44,45}$

Release Assays. The nanogel (labeled with FITC) release from the oDex-nanogel hydrogels was evaluated by fluorimetry (Figure 9B). The nanogel was gradually released over time, paralleling the hydrogel degradation; the total fraction of nanogel released reached 75 and 54\% for oDex-nanogel $1 \mathrm{mg} / \mathrm{mL}$ and oDex-nanogel $3 \mathrm{mg} / \mathrm{mL}$, respectively, after 35 days. Although a significant mass loss of the hydrogel is observed in the first few days, a steady and continuous release of nanogel is observed in an expanded time frame, up to 4 weeks.

Lynch and Dawson ${ }^{16,17}$ have also described a composite material, composed of poly( $N$-isopropylacrylamide) (NIPAM)based microgel particles entrapped in a hydrogel matrix. They called this composite material the "plum-pudding gel". The release of two compositionally different solutes from this composite hydrogel composed of two different populations of microgel particles embedded in a single bulk matrix was described, showing the potential of the plum-pudding gel as a multifunctional platform for controlled release. Later on, the same group studied the controlled release of fluvastatin from the plum-pudding gel. ${ }^{14}$

These composite materials present themselves as an alternative approach to "simply" incorporate drugs into polymer nano or microgels. This strategy has some advantages comparatively to those of drug-loaded polymeric particles: by separating the functional role (of the polymeric particles) from the support matrix (polymeric hydrogel), it can simultaneously release several different drugs; additionally, elution of the polymeric particles does not degrade the polymer hydrogel, which leads to a more stable long-term elution profile. So, this composite oDex-nanogel hydrogel could be useful to overcome the initial burst release phenomenon often observed in nanogel and hydrogel drug delivery systems because it allows for the nanogel slow and controlled release. Preliminary assays were carried out to study the oDex-nanogel hydrogel as a controlled release system for proteins.

Figure 10 shows the percentage of rIL-10 released from oDex and oDex-nanogel hydrogels in PBS with $10 \%$ FBS. The

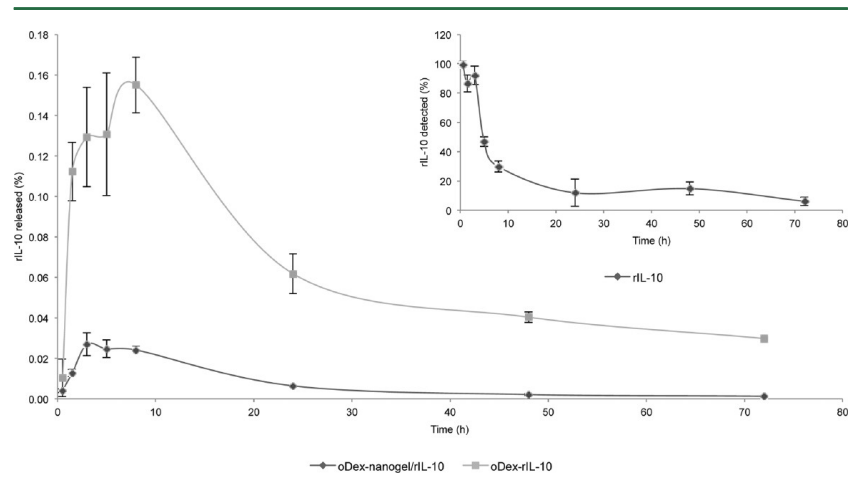

Figure 10. rIL-10 release from oDex-rIL-10 and oDex-nanogel/rIL-10 hydrogels. Inset shows the percentage of the initial soluble rIL-10 remaining detectable using the ELISA under the same conditions as in the release assays. Shown are mean \pm SD values $(n=3)$. general goal of this experiment was compromised by the low stability of rIL-10. rIL-10 is a rather unstable protein, as can be seen on the inset of Figure 10, where it is observed that the amount of solubilized (nonencapsulated) rIL-10 detectable with the ELISA decreases significantly and quickly, probably due to denaturation and aggregation. The stability of rIL-10 was accessed at $37{ }^{\circ} \mathrm{C}$ by recording the CD spectra over time. rIL10 , at time 0 , presented a typical CD spectrum of a helical protein, as expected. ${ }^{46-48}$ The analysis of the mean residual ellipticity $(\theta)$ variation, at $222 \mathrm{~nm}$, allowed the prediction of the rIL-10 half life, and this sample showed a half life of $4.4 \pm$ 0.7 days corroborating the high instability demonstrated by the protein in this release assay.

Despite rIL-10 improved stability when encapsulated in the nanogel (as it has been previously demonstrated ${ }^{13}$ ), its release from the hydrogels (oDex and oDex-nanogel) is observed in both cases only in the first 6-10 h of the experiment. Actually, the amount detected in the supernatant decreased henceforward, due to faster aggregation as compared with fresh cytokine being released from the hydrogel. It can nevertheless be remarked that the release of the protein occurs at low pace when encapsulated in the nanogel, which thus seems to offer an additional control on the release kinetics.

Therefore, more stable protein was selected to evaluate the performance of the hydrogel-nanogel as a controlled protein release system. The Ins-FITC release from oDex and oDexnanogel hydrogels, in PBS, was estimated by fluorimetry and is displayed in Figure 11. The release profile observed was similar

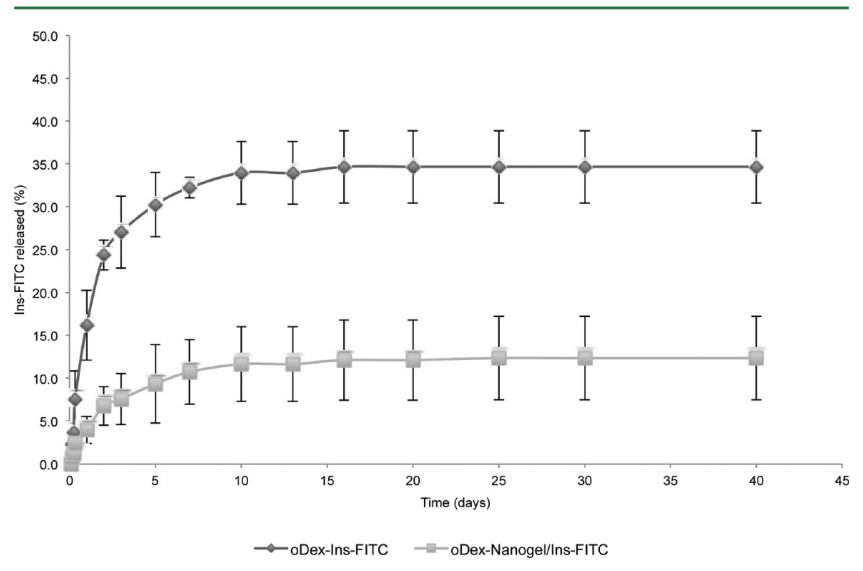

Figure 11. Ins-FITC release from oDex-Ins-FITC and oDex-nanogel/ Ins-FITC hydrogels. Shown are mean \pm SD values $(n=3)$.

in both situations: the protein is released in a slow and sustained way for at least 40 days. However, because the incorporation of the dextrin nanogel in the oDex hydrogel offered an additional barrier for the release, the percentage of Ins-FITC detectable was significantly lower when the oDex-nanogel was used, as expected. It was also noticed that the protein did not rapidly diffuse out of the oDex hydrogel. On the contrary, the amount of Ins-FITC released over time was rather low compared with other studies where different hydrogel and loading methods are used. ${ }^{49,50}$

Protein loading into, and release from, a hydrogel are controlled by various factors including: the pore volume fraction; the pore sizes and their interconnections; the size of the protein; and also the interactions between the protein and polymer chains. Specifically, the protein-polymer interactions not only affect protein loading capacity and release kinetics but also greatly influence the protein release mechanisms. ${ }^{31,51,52}$ 
In some studies, proteins and other drugs are loaded by immersing lyophilized hydrogels into drug solutions, and this generally translates into to the rapid diffusion of the drugs throughout the pores of the hydrogels. ${ }^{49,50}$ Here the oDex was dissolved in the protein solution before $\mathrm{ADH}$ addition, and this allows the spontaneous chemical reaction between the reactive aldehydes present on the oDex and the amines of the protein, forming a stable oDex-Ins conjugate before gelation. Because of this conjugation, the Ins release from the oDex hydrogels do not occur simply by diffusion. A lower degree of oDex-Ins conjugation can be obtained by adding Ins and $\mathrm{ADH}$ simultaneously. Under these conditions, $\sim 77 \%$ Ins is released after 6 days. It should be remarked that the oDex-Ins conjugates might play an additional feature for the control of the protein release system. Recently, a polymer-protein conjugate was described as a bioresponsive polymer therapeutic to promote wound healing. ${ }^{31,52}$ This method was based on the conjugation of dextrin with a recombinant human epidermal growth factor (rhEGF) and the observation that $\alpha$-amylase, by degrading dextrin, allowed the slow release of biologically active rhEGF. Accordingly, in an in vivo situation, the Ins release from the oDex hydrogels will occur, not only by diffusion but also as a result of dextrin degradation by the $\alpha$-amylases present in the blood. Therefore, this system may constitute a sophisticated delivery system of which potential will be further evaluated in vivo, namely, assessing the biocompatibility and protein release. The contribution of amylases (in vitro and in vivo) for the protein release will be accessed. Furthermore, the potential of the incorporated nanogels for the encapsulation of poorly water-soluble low-molecular-weight molecules will also be analyzed.

\section{CONCLUSIONS}

In this study, oDex and oDex-nanogel hydrogels were developed for the sustained delivery of therapeutical proteins. The oDex hydrogels presented acceptable mechanical properties and revealed an overall good biocompatibility. The oDex and oDexnanogel hydrogels were biodegradable, with a macroporous structure, and allowed for the nanogel release over time.

rIL-10 and Ins-FITC were loaded in both hydrogels (oDex and oDex-nanogel), and their release was evaluated. rIL-10 studies were compromised by the very low stability of the protein. Ins-FITC was not completely released from oDex hydrogel due to protein conjugation, and the presence of the nanogel in the oDex-nanogel offered an additional resistance to the Ins-FITC release translated by the lower amount of InsFITC detected over time.

The straightforward preparation and the controllable release properties of oDex hydrogels make them attractive for the design of injectable protein delivery systems. The presence of a dispersed hydrophobic phase in the hydrogel may represent an important advantage of the newly developed material. The longer sustained release of the protein is advantageous in these kinds of applications because it may provide a continuous delivery of protein and prevent problems of cyclic variations in the protein concentration in blood with time and offer a maximum pharmacological efficiency at a minimum drug dose, reducing administration frequency and improving patient compliance to the therapy.

\section{AUTHOR INFORMATION}

\section{Corresponding Author}

*E-mail: fmgama@deb.uminho.pt. Tel: (+351) 253604418. Fax: (+351) 253678986 .

\section{Author Contributions}

${ }^{\dagger}$ These authors contributed equally to this study.

Notes

The authors declare no competing financial interest.

\section{ACKNOWLEDGMENTS}

V.C. and D.S. were supported by the grants SFRH/BD/27359/ 2006 and SFRH/BD/64571/2009, respectively, from Fundação para a Ciência e Tecnologia (FCT), Portugal. We thank FCT funding through PTDC and COMPETE.

\section{REFERENCES}

(1) Haag, R.; Kratz, F. Angew. Chem., Int. Ed. 2006, 45, 1198-215.

(2) Branco, M. C.; Schneider, J. P. Acta Biomater. 2009, 5, 817-31.

(3) Nayak, S.; Lyon, L. A. Angew. Chem., Int. Ed. 2005, 44, 7686-708.

(4) Devalapally, H.; Chakilam, A.; Amiji, M. M. J. Pharm. Sci. 2007, 96, 2547-65.

(5) Lee, K. Y.; Mooney, D. J. Chem. Rev. 2001, 101, 1869-1879.

(6) Hoare, T. R.; Kohane, D. S. Polymer 2008, 49, 1993-2007.

(7) Frampton, J. E.; Plosker, G. L. Drugs 2003, 63, 2079-105.

(8) Hreczuk-Hirst, D.; Chicco, D.; German, L.; Duncan, R. Int. J. Pharm. 2001, 230, 57-66.

(9) Carvalho, J.; Goncalves, C.; Gil, A. M.; Gama, F. M. Eur. Polym. J. 2007, 43, 3050-3059.

(10) Carvalho, J.; Moreira, S.; Maia, J.; Gama, F. M. J. Biomed. Mater. Res., Part A 2010, 93, 389-99.

(11) Goncalves, C.; Gama, F. M. Eur. Polym. J. 2008, 44, 3529-3534.

(12) Goncalves, C.; Martins, J. A.; Gama, F. M. Biomacromolecules 2007, 8, 392-8.

(13) Carvalho, V.; Castanheira, P.; Faria, T. Q.; Goncalves, C.; Madureira, P.; Faro, C.; Domingues, L.; Brito, R. M. M.; Vilanova, M.; Gama, F. M. Int. J. Pharm. 2010, 400, 234-242.

(14) McGillicuddy, F. C.; Lynch, I.; Rochev, Y. A.; Burke, M.; Dawson, K. A.; Gallagher, W. M.; Keenan, A. K. J. Biomed. Mater. Res., Part A 2006, 79A, 923-933.

(15) Salvati, A.; Soderman, O.; Lynch, I. J. Phys. Chem. B 2007, 111, $7367-7376$

(16) Lynch, I.; Dawson, K. A. J. Phys. Chem. B 2003, 107, 96299637.

(17) Lynch, I.; de Gregorio, P.; Dawson, K. A. J. Phys. Chem. B 2005, 109, 6257-6261.

(18) Bouhadir, K. H.; Hausman, D. S.; Mooney, D. J. Polymer 1999, 40, 3575-3584.

(19) Mocellin, S.; Panelli, M. C.; Wang, E.; Nagorsen, D.; Marincola, F. M. Trends Immunol. 2003, 24, 36-43.

(20) Asadullah, K.; Sterry, W.; Volk, H. D. Pharmacol. Rev. 2003, 55, 241-269.

(21) Duckworth, W. C.; Fawcett, J.; Tsui, B. T.; Bennett, R. G.; Hamel, F. G. Biochem. Biophys. Res. Commun. 2004, 318, 1019-1024.

(22) Zhang, Y. Q.; Ma, Y.; Xia, Y. Y.; Shen, W. D.; Mao, J. P.; Xue, R. Y. J. Controlled Release 2006, 115, 307-315.

(23) Jia, X. Q.; Burdick, J. A.; Kobler, J.; Clifton, R. J.; Rosowski, J. J.; Zeitels, S. M.; Langer, R. Macromolecules 2004, 37, 3239-3248.

(24) Maia, J.; Ribeiro, M. P.; Ventura, C.; Carvalho, R. A.; Correia, I. J.; Gil, M. H. Acta Biomater. 2009, 5, 1948-1955.

(25) Maia, J.; Ferreira, L.; Carvalho, R.; Ramos, M. A.; Gil, M. H. Polymer 2005, 46, 9604-9614.

(26) Maia, J.; Carvalho, R. A.; Coelho, J. F. J.; Simoes, P. N.; Gil, M. H. Polymer 2011, 52, 258-265.

(27) Kristiansen, K. A.; Potthast, A.; Christensen, B. E. Carbohydr. Res. 2010, 345, 1264-71.

(28) Aalmo, K. M.; Painter, T. J. Carbohydr. Res. 1981, 89, 73-82. 
(29) Li, J.; Wan, Y. Z.; Li, L. F.; Liang, H.; Wang, J. H. Mater. Sci. Eng., C 2009, 29, 1635-1642.

(30) Moreira, S.; da Costa, R. M. G.; Guardao, L.; Gartner, F.; Vilanova, M.; Gama, M. J. Bioact. Compat. Polym. 2010, 25, 141-153.

(31) Hardwicke, J.; Ferguson, E. L.; Moseley, R.; Stephens, P.; Thomas, D. W.; Duncan, R. J. Controlled Release 2008, 130, 275-283.

(32) Anseth, K. S.; Bowman, C. N.; BrannonPeppas, L. Biomaterials 1996, 17, 1647-1657.

(33) Massia, S. P.; Stark, J. J. Biomed. Mater. Res. 2001, 56, 390-399.

(34) Ferreira, L.; Rafael, A.; Lamghari, M.; Barbosa, M. A.; Gil,

M. H.; Cabrita, A. M. S.; Dordick, J. S. J. Biomed. Mater. Res., Part A

2004, 68A, 584-596.

(35) Chen, Y. M.; Shiraishi, N.; Satokawa, H.; Kakugo, A.; Narita, T.; Gong, J. P.; Osada, Y.; Yamamoto, K.; Ando, J. Biomaterials 2005, 26, 4588-96.

(36) Schneider, G. B.; English, A.; Abraham, M.; Zaharias, R.; Stanford, C.; Keller, J. Biomaterials 2004, 25, 3023-8.

(37) Gonçalves, C.; Gama, F. M., Eur. Polym. J. 2008, 44.

(38) Goncalves, C.; Torrado, E.; Martins, T.; Pereira, P.; Pedrosa, J.; Gama, M. Colloids Surf., B 2010, 75, 483-489.

(39) Gonçalves, C.; Pereira, P.; Schellenberg, P.; Coutinho, P. J. G.; Gama, F. M. J. Biomater. Nanobiotechnol. 2011.

(40) Zhang, X. Z.; Lewis, P. J.; Chu, C. C. Biomaterials 2005, 26, 3299-3309.

(41) Nuño-Donlucas, S. M.; Sanches-Diaz, J. C.; Rabelero, M.; Cortes-Ortega, J.; Luhrs-Olmos, C. C.; Fernandez-Escamilla, V. V.; Mendizabal, E.; Puig, J. E. J. Colloid Interface Sci. 2004, 270, 94-98.

(42) Puig, L. J.; Sanchez-Diaz, J. C.; Villacampa, M.; Mendizabal, E.; Puig, J. E.; Aguiar, A.; Katime, I. J. Colloid Interface Sci. 2001, 235, 278-282.

(43) Pillai, O.; Panchagnula, R. Curr. Opin. Chem. Biol. 2001, 5, 447451.

(44) Peppas, N. A.; Bures, P.; Leobandung, W.; Ichikawa, H. Eur. J. Pharm. Biopharm. 2000, 50, 27-46.

(45) Jen, A. C.; Wake, M. C.; Mikos, A. G. Biotechnol. Bioeng. 1996, 50, 357-364.

(46) Josephson, K.; DiGiacomo, R.; Indelicato, S. R.; Ayo, A. H.; Nagabhushan, T. L.; Parker, M. H.; Walter, M. R. J. Biol. Chem. 2000, $275,13552-13557$.

(47) Zdanov, A.; Schalk-Hihi, C.; Gustchina, A.; Tsang, M.; Weatherbee, J.; Wlodawer, A. Structure 1995, 3, 591-601.

(48) Zdanov, A.; Schalk-Hihi, C.; Wlodawer, A. Protein Sci. 1996, 5, 1955-62.

(49) Hirakura, T.; Yasugi, K.; Nemoto, T.; Sato, M.; Shimoboji, T.; Aso, Y.; Morimoto, N.; Akiyoshi, K. J. Controlled Release 2010, 142, 483-489.

(50) Akiyoshi, K.; Morimoto, N.; Endo, T.; Iwasaki, Y. Biomacromolecules 2005, 6, 1829-1834.

(51) Yang, Y. Y.; Wu, J. Y.; Liu, S. Q.; Heng, P. W. S. J. Controlled Release 2005, 102, 361-372.

(52) Moseley, R.; Hardwicke, J. T.; Hart, J.; Bell, A.; Duncan, R.; Thomas, D. W. J. Controlled Release 2011, 152, 411-417. 